\title{
Quantitative Methods for Analyzing Intimate Partner Violence in Microblogs: Observational Study
}

Christopher Michael Homan $^{1 *}$, BA, MSc, PhD; J Nicolas Schrading ${ }^{1 *}, \mathrm{BSc}, \mathrm{MSc}$; Raymond W Ptucha ${ }^{1 *}$, BSc, MSc, $\mathrm{PhD}$; Catherine Cerulli2 ${ }^{2 *}$, BA, JD, PhD; Cecilia Ovesdotter Alm ${ }^{1 *}$, BSc, MSc, PhD

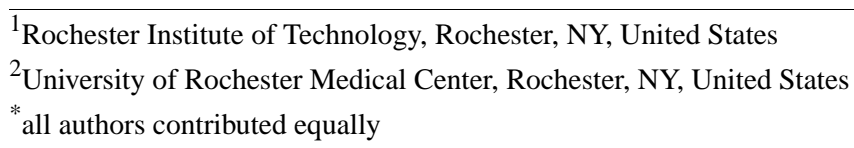

Corresponding Author:

Christopher Michael Homan, BA, MSc, PhD

Rochester Institute of Technology

102 Lomb Memorial Dr

Rochester, NY, 14623-5608

United States

Phone: 15857196835

Email: $\underline{\mathrm{cmh} @ c s . r i t . e d u}$

\section{Abstract}

Background: Social media is a rich, virtually untapped source of data on the dynamics of intimate partner violence, one that is both global in scale and intimate in detail.

Objective: The aim of this study is to use machine learning and other computational methods to analyze social media data for the reasons victims give for staying in or leaving abusive relationships.

Methods: Human annotation, part-of-speech tagging, and machine learning predictive models, including support vector machines, were used on a Twitter data set of 8767 \#WhyIStayed and \#WhyILeft tweets each.

Results: Our methods explored whether we can analyze micronarratives that include details about victims, abusers, and other stakeholders, the actions that constitute abuse, and how the stakeholders respond.

Conclusions: Our findings are consistent across various machine learning methods, which correspond to observations in the clinical literature, and affirm the relevance of natural language processing and machine learning for exploring issues of societal importance in social media.

(J Med Internet Res 2020;22(11):e15347) doi: $\underline{10.2196 / 15347}$

\section{KEYWORDS}

intimate partner violence; social media; natural language processing

\section{Introduction}

Intimate partner violence (IPV) encompasses physical violence, sexual violence, stalking, and psychological aggression (including coercive acts) by a current or former intimate partner [1]. The mental and physical consequences of IPV include depression, posttraumatic stress disorder, and suicidal thoughts and behaviors [2]. Physical consequences can include myriad acute and chronic health conditions, including but not limited to functional health status, cardiac health, complicated sleep histories, and higher reports of chronic pain [1,3-5]. These effects are long-lasting [1], and IPV affects people regardless of their sexual orientation or gender identity [6]. How IPV impacts any particular individual may depend on their childhood or adolescent experiences [7] or socioeconomic class [8]. For instance, women tend to be injured more severely and are killed more frequently than their male counterparts [8]. In the United States, $6.4 \%$ of men and $6.6 \%$ of women are affected by physical violence, sexual violence, or stalking annually [8]. Despite the similarity in frequencies between the genders, there are differences regarding severity and mortality, with more women reporting severe injury and dying as a result of IPV-related deaths.

A major gap in knowledge on the prevalence of IPV exists because population-level data are difficult to collect, particularly from victims [9]. Consequently, theories about why people become involved and remain in abusive relationships are based 
primarily on qualitative studies and surveys with small samples, or larger samples of individuals who are often in the process of help-seeking. We know less about why people leave abusive partners because the process is often out of the vision of traditional service-providing agencies.

An alternate source of quantifiable data, such as Facebook, Twitter, or Instagram, is an alternate source of quantifiable data. It provides textual narratives at a level of personal detail reminiscent of focus groups or one-on-one interviews, but over populations larger than nearly any survey. Such an unsurpassed combination of scale and detail promises great rewards to the social, behavioral, and health sciences. Although these narrative tweets are short in length, they are a potentially rich source of information about IPV. Moreover, microblogging platforms such as Twitter offer a potential venue for public health prevention messaging that can be accessed broadly.

The amount of data present in social media is too great for human IPV experts to inspect manually. However, its semistructured, qualitative nature, along with the informality of the language used, means that human judgment is needed to make sense of the data. Machine learning has been used in many other settings to bridge this gap, and it can perform certain specialized tasks nearly as well as humans [10].

In this study, we present a machine learning workflow for social media data to hear, on a national, population-level scale, from victims themselves on why they stayed with abusive partners, or what helped them to leave. As a case study, we use this workflow to perform a comparative study on Twitter data tagged by \#WhyIStayed or \#WhyILeft. Such hashtags provide prelabeled data for machine learning.

Given the opportunistic nature of hashtagging, it is helpful to understand the history of these particular tags. On February 15, 2014 Ray Rice, a famous professional athlete in the National
Football League, was arrested to assault his then fiancée (now wife), Janay Rice (née Palmer) [11]. First, news and sports media downplayed the incident. However, on February 19th, a video of the incident surfaced that showed Rice assaulting his fiancé in an elevator and eventually dragging her limp body out by her shoulders [11]. This caused an uproar in the advocacy and sports communities, and a backlash against the victim [11] (the backlash escalated after they married, one day after he was indicted by a grand jury). In response, on September 8, 2014 activist Beverly Gooden began using the hashtag \#WhyIStayed to encourage victims of abuse to tell their stories about what kept them in abusive relationships; \#WhyILeft appeared soon thereafter [12]. Tweets carrying these hashtags soon numbered in the tens of thousands, as people around the world decided to share their stories of IPV.

As an organizing principle and example of how to make sense out of the kind of opportunities for understanding and sensemaking that such movements provide, we focus on the specific question: Why did both \#WhyIStayed and \#WhyILeft go viral? Certainly, tweets disclosing stories about staying or leaving could be framed in terms of either hashtag, and adopting one or the other alone might have benefited the movement with a single (and thus easier-to-recall) catchphrase. These hashtags were invented by victims of IPV and virally adopted by a large community of victims over a relatively brief period of time. See Figure 1. Thus, they represent, among other things, victims of IPV not just telling their stories, but doing so in their own terms, rather than in those of health providers, researchers, or the criminal justice system. In a seminal work exploring 32 in-depth interviews with victims, Lempert [13] suggests that telling is a significant step in seeking help because it makes public their fictions of intimacy (citing [14]). Our primary goal was to assess how information gleaned through Twitter data could inform our understanding of survivors' lived experiences with staying in, or leaving, abusive relationships.

Figure 1. Counts per hour of \#WhyIStayed (dotted) or \#WhyILeft (solid) tweets from 9/8 to 9/12. Times in Eastern Standard Time, vertical lines mark 12-hour periods, with label corresponding to its left line. We removed spam from this set, but not meta tweets.

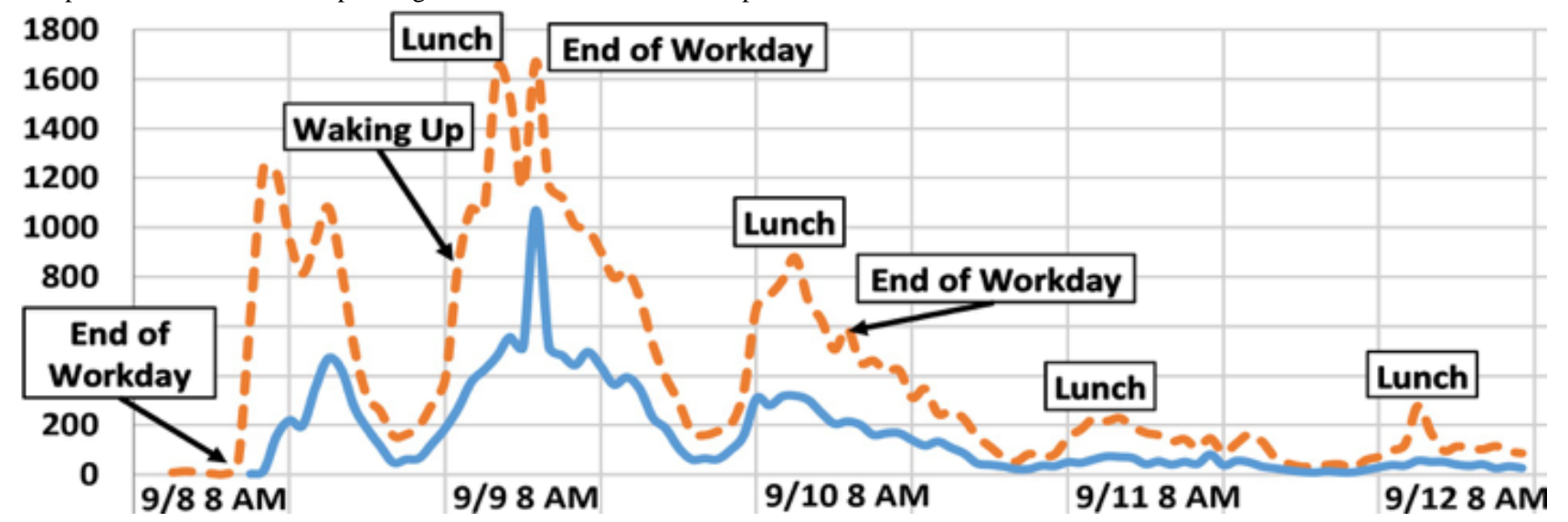

\section{Methods}

\section{Data}

We collected a corpus of tweets containing the hashtag \#WhyIStayed or \#WhyILeft using the Twitter and Topsy application programming interfaces, of which the latter is currently defunct. This corpus spans the beginning of September (the start of the trend) to the beginning of October 2014, when volume dropped to background levels. The majority of tweets are from the first week of the trend's creation (Figure 1).

\section{Preprocessing}

To partially anonymize the data, we replaced all URLs with the generic token "url." We removed spam tweets based on the 
usernames of prominent spammers and key spam hashtags such as \#MTVEMA, \#AppleWatch, and \#CMWorld. Additionally, we removed tweets in which Twitter accounts for DiGiorno Pizza tweeted \#WhylStayed You had pizza. Therefore, we excluded tweets containing tokens pizza or digiorno. The resulting corpus contained over 57,000 unique tweets.

Many tweets in the dataset were reflections on the trend itself or contained messages of support to those who shared their stories of abuse, for example, not usually a fan of hashtag trends, but \#WhyIStayed is incredibly powerful. \#NFL \#RayRice. These instances, here denoted meta-tweets, were often retweeted, but they rarely contained reasons for staying or leaving (which were the interests of this study), so we excluded any tweets containing the keywords janay/ray rice, football, tweets, trend, and video.

\section{Extracting Hashtag Labels}

Typically, tweets disclosed reasons for staying (, respectively, leaving) and were prepended or appended with the hashtags \#WhyIStayed (, respectively, \#WhyILeft). \#WhyILeft because I gained the courage to love myself. If the tweet contained only one of the target hashtags, the instance was labeled with that hashtag. For tweets marked by both hashtags, we split them into two identical instances, each distinctly labeled with one of the hashtags.
The resulting corpus comprised 24,861 \#WhyIStayed and 8767 \#WhyILeft labeled instances. This hashtag class imbalance may be a consequence of the origins and media portrayals of the trend (the tweet that started the trend contained only the hashtag \#WhyIStayed, and media reports tended to refer to the "\#WhyIStayed" phenomenon) rather than an indicator that more victims stay than leave. The first \#WhyILeft tweet occurred hours after the first \#WhyIStayed tweet, and never gained as much traction (Figure 1)

To normalize comparisons between the tweets associated with each of these hashtags, we randomly sampled from the \#WhyIStayed tweets to obtain a balanced set of 8767 examples per class. Of the 8767, we held out 1315 (14.99\%) of this balanced set as a final test set for our machine learning experiments, and left the remaining $7452(85.00 \%)$ as the devset (all of the remaining analysis in this section used the devset).

Manual inspection of the devset tweets revealed that, in addition to telling stories of IPV, the tweets served other purposes. To gain insight into the coarse grained language of these remaining tweets, we randomly sampled 1000 of them from the devset (473/1000, 47.30\% \#WhyIStayed and 527/1000, 52.70\% \#WhyILeft) and annotated them according to the coding scheme shown in Table 1, that is, as advertisements, jokes, tweets about leaving, meta (ie, tweets discussing or reporting on the \#WhyIStayed/\#WhyILeft phenomenon), tweets about staying, or other.

Table 1. Summary of labels from all four annotators, A1 through A4, compared with the gold standard. Each cell indicates the number of tweets an annotator gave a label to.

\begin{tabular}{|c|c|c|c|c|c|c|c|}
\hline Annotator & Ads & Jokes & Leave & Meta & Other & Stay & Total \\
\hline \multicolumn{8}{|l|}{ A1 } \\
\hline $\mathrm{L}^{\mathrm{a}}$ & 3 & 6 & 356 & 57 & 67 & 38 & 527 \\
\hline$S^{b}$ & 6 & 12 & 28 & 97 & 31 & 299 & 473 \\
\hline \multicolumn{8}{|l|}{ A2 } \\
\hline $\mathrm{L}$ & 10 & 6 & 378 & 33 & 47 & 53 & 527 \\
\hline$S$ & 13 & 6 & 33 & 74 & 47 & 300 & 473 \\
\hline \multicolumn{8}{|l|}{$\mathbf{A 3}$} \\
\hline $\mathrm{L}$ & 2 & 10 & 405 & 49 & 2 & 59 & 527 \\
\hline$S$ & 7 & 18 & 29 & 97 & 1 & 321 & 473 \\
\hline \multicolumn{8}{|l|}{$A 4^{\mathrm{c}}$} \\
\hline $\mathrm{L}$ & 3 & 1 & 122 & 19 & 15 & 14 & 174 \\
\hline$S$ & 3 & 0 & 15 & 35 & 14 & 92 & 159 \\
\hline
\end{tabular}

\footnotetext{
${ }^{\mathrm{a}} \mathrm{L}$ : \#Why I Left.

${ }^{\mathrm{b}} \mathrm{S}$ : \#Why I Stayed.

${ }^{\mathrm{c}} \mathrm{A} 4$ annotated only the first 333 tweets.
}

Before commencing annotation, to help better understand the distinct roles of each of the two hashtags played, we removed all occurrences of them from the tweets, to see if annotators could infer from the rest of the language whether the tweet was about staying or leaving without having the hashtag as a cue. We then studied the differences between tweets about staying versus leaving in terms of ngram bag-of-word models as features, part-of-speech tags, and a restriction to subject-verb-object tweets only. 


\section{Machine Learning on Linguistic Features}

One way to address our main research question is to ask whether \#WhyIStayed and \#WhyILeft hashtags indicated distinct classes of micronarratives or were merely framing devices. To address this question, we trained naïve Bayes, linear support vector machine (SVM), and radial basis function (RBF) SVM classifiers from the scikit-learn python library [15], using the hashtags as ground truth, and various language features as input. We then use the SVMs to report the features that have the most predictive power. We also considered neural models [16,17], but it was harder to make sense of these results.

\section{Results}

\section{Human Annotation}

Four of the authors of this paper performed the annotation task. The overall agreement overlap was 0.77. Randolph's free-marginal multirater kappa [18] score was 0.72 . We chose this multirater kappa because it allows any distribution of class labels that annotators assign (ie, it is free-marginal), unlike Fleiss' multirater kappa, which assumes a predetermined distribution [18]. According to the resulting annotations (Table 1), on average (over all annotators), $35.28 \%$ (1176/3333) of the instances were reasons for staying (S), 40.98\% (1366/3333) were reasons for leaving (L), $13.83 \%$ (461/3333) were meta comments, $1.77 \%$ (59/3333) were jokes, $1.41 \%$ (47/3333) were ads, and $6.72 \%(224 / 3333)$ did not match prior categories (other).
The limited contextual information that such tweets provided sometimes made it difficult to interpret unambiguously. For instance because $i$ was slowly dying anyway was marked as $\mathrm{S}$ by two annotators and L by the other two. The annotators disagreed on whether the victim decided to stay out of a sense of resignation or left because they felt there was nothing left to lose. (The ground truth label is \#WhyILeft.) Another example of disagreement was two years of bliss, followed by uncertainty and fear. (This tweet's label is \#WhyIStayed.) However, our results show that most tweets contained enough information for humans to infer their original hashtag labels, with annotators correctly identifying $\mathrm{L}$ more frequently than $\mathrm{S}$.

\section{Lexical Usage}

Basic lexical statistics in the balanced devset before lowercasing, stoplisting, and lemmatizing are shown in Table 2. The top nine most frequent unigrams, bigrams, and trigrams of words in the balanced dataset after lowercasing, stoplisting, and lemmatizing are shown in Tables 3 and 4. In order for word and ngram counts to reflect the words representing content rather than the words serving grammatical functions or tweet markup, before making these counts, we stoplisted, lemmatized, and excluded start-of-sentence and end-of-sentence tokens from each tweet.

Each table reveals new insights into the \#WhyIStayed/\#WhyILeft movement, so we examine each in turn.

Table 2. Basic lexical statistics on the tokens and types in the two balanced sets. Types are unique tokens whereas hapax legomena are those tokens that only occur once in the data set.

\begin{tabular}{lll}
\hline Parameters & \#WhyIStayed & \#WhyILeft \\
\hline Number of tokens & 130,545 & 118,768 \\
Number of types & 7094 & 6269 \\
Type:token ratio & 0.054 & 0.053 \\
Number of hapax legomena & 3871 & 3340 \\
\hline
\end{tabular}

Table 3. Top 9 most frequent unigrams (left) and bigrams (right) after preprocessing, with their respective frequencies in the Twitter devset.

\begin{tabular}{|c|c|c|c|c|c|c|c|}
\hline \multicolumn{4}{|l|}{ Unigrams } & \multicolumn{4}{|l|}{ Bigrams } \\
\hline \#WhyIStayed & Frequency, n & \#WhyILeft & Frequency, n & \#WhyIStayed & Frequency, n & \#WhyILeft & Frequency, n \\
\hline think & 1061 & love & 930 & think love & 127 & deserve better & 298 \\
\hline love & 971 & realize & 888 & abusive relationship & 112 & finally realize & 103 \\
\hline leave & 872 & want & 702 & feel like & 95 & realize deserve & 80 \\
\hline believe & 578 & know & 594 & try leave & 78 & want live & 66 \\
\hline tell & 550 & better & 570 & emotional abuse & 72 & learn love & 61 \\
\hline want & 540 & deserve & 558 & think deserve & 67 & want daughter & 59 \\
\hline say & 529 & abuse & 507 & make believe & 64 & year old & 56 \\
\hline
\end{tabular}


Table 4. Top 9 most frequent trigrams after preprocessing, with their respective frequencies in the Twitter devset. The number in each cell indicates the number of times the trigram appeared in the dataset.

\begin{tabular}{llll}
\hline \#WhyIStayed & Frequency, $\mathrm{n}$ & \#WhyILeft & Frequency, $\mathrm{n}$ \\
\hline make feel like & 37 & realize deserve better & 56 \\
pregnant hit url & 25 & know deserve better & 40 \\
stay abusive relationship & 25 & finally realize deserve & 19 \\
change conversation url & 22 & son deserve better & 18 \\
leave man yell & 21 & true love hurt & 18 \\
abusive relationship url & 20 & daughter deserve better & 17 \\
man yell url & 20 & want daughter think & 15 \\
say kill leave & 20 & want daughter grow & 15 \\
church support spousal & 19 & daughter grow think & 15 \\
\hline
\end{tabular}

\section{Unigrams}

The six words appearing in both columns of the unigram table (love, leave, abuse, want, and know) reveal common themes shared by both hashtags (Table 3). Our bi- and tri- gram analyses (presented below) reveal differences in how these common words are used. However, from the unigram perspective, the commonality of these words suggests, in the case of leave and abuse, that both \#WhyIStayed and \#WhyILeft tweets are often framed in terms of a leaving event, and that both often acknowledge the abuse that happened. This is perhaps not surprising, as the tags themselves suggest looking back on past abusive relationships. Love and want reveal that strong emotional forces are associated with both hashtags. Know indicates that knowledge (or lack thereof) is associated with both hashtags.

Of the words found in one list only, think and believe in the \#Stayed list and realize in the \#Left list suggest a transition between staying and leaving that involves learning the truth about false beliefs. The presence of tell and say in the \#Stayed list suggests that an abuser coerced, deceived, or in some other way, emotionally manipulated the narrator. Deserve and life in the \#Left list express positive sentiments.

\section{Bigrams}

Deserve better - the most common bigram by far-indicates that a change in one's sense of self dignity or fairness plays a major role in leaving. Both realize and deserve occur three times in the \#Left list, suggesting that increased awareness, particularly from the perspective of justice or fairness, were important forces in helping narrators leave abusive relationships (Table 3).

Two words (love and deserve) appear in both lists. Each is paired with think in \#Stayed and realized in \#Left, suggesting a change in awareness of what love and fair treatment really are, respectively. That deserve also appears with know for \#Left suggests determination.

The presence of emotional abuse in the \#Stayed list confirms that, to the narrators of these tweets, emotional manipulation played a role in their staying in abusive relationships.
One telling bigram pairing is kill leave from the \#Stayed list and want live from the \#Left list. They indicate that people both remained and left abusive relationships out of fear for the safety of self and loved ones (the latter being supported by considerations about children, such as year old, want daughter, in the \#Left list).

\section{Trigrams}

The most frequent trigrams in each list make feel like and realize deserve better, respectively, make an interesting pair. They seem to indicate the important role emotional manipulation plays in staying, and that leaving is precipitated by a realization or epiphany, perhaps sometimes ones that break the spell of emotional abuse (Table 4).

Hit, yell, and kill all appear in the \#WhyIStayed list, suggesting that violence is an important force in keeping people in abusive relationships. Notably, man appears twice in this list. The trigram church support spousal shows the important role institutions and moral or religious values play.

Better, deserve, and daughter each appear four times in the \#WhyILeft list. This shows that concerns about the welfare of dependents (note that son also appears on the list) or a desire for a better life (and that such a life is deserved) drive decisions to leave. The most common word in the \#Stayed list, url (which appears four times), is harder to interpret; recall that, to protect privacy, we removed all URLs and replaced them with this token.

\section{Subject-Verb-Object Structures}

Fourteen percent of the dev- and test-set tweets have a subject-verb-object (SVO) structure, in which (a) the abuser is doing something to the victim, or (b) the victim is explaining something about the abuser or self. Such SVO structures represent the largest proportion of the total number of dependency structures in this data. Thus, we focus on its exploration in both corpus analytics and automated classification. These SVO structures provide insight into the abuser-victim relationship while maintaining sentence-level structures large enough to convey or indicate syntactic relationships, which tend to be more interpretable than isolated words. 
We used the following conditional model to identify the most indicative verbs in terms of predicting hashtag class (\#WhyIStayed vs \#WhyILeft), among SVO tweets. Starting at the lemmatized predicate verb in each dependency parse, whenever the predicate verb followed an abuser subject word and preceded a victim object word, we added it to a distribution conditioned on class. The abuser subject words were he, $b f$, boyfriend, father, dad, husband, brother, and man for a male abuser, she, gf, girlfriend, mother, mom, wife, sister, and woman for a female abuser, and finally, pastor, abuser, offender, ex, $x$, lover, church, and they were used as neutral references. The victim object words were me, sister, brother, child, kid, baby, friend, her, him, man, and woman. These are denoted here as abusers onto victim structures. Analogous methods were used to extract structures in which the victim was the subject. We then determined the most indicative verb predicates from these conditional frequency distributions using the following formula for each such predicate:

$$
\text { ratio }=\frac{\max \left\{\text { count }_{\text {left }}, \text { count }_{\text {stayed }}\right\}}{\text { count }_{\text {left }}+\text { count }_{\text {stayed }}}
$$

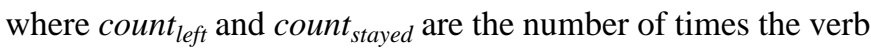
appears in \#WhyILeft or \#WhyIStayed tweets, respectively. Table 5 shows those where the ratio is greater than 0.70 and, to avoid a bias toward lower frequency verbs, the total count exceeds a threshold of $0.5 \%$ of the total number of instances. In the case of abuser onto victim, the resulting frequency threshold was 11, and in the victim as subject, it was 68 .

Table 5. The most indicative (in the direction of staying) verbs for abuser onto victim and victim as subject in the tweets having subject verb object structures. An exclamation point (!) before a verb indicates negation (eg, the phrase he did not love me would give the verb !love). Each cell indicates the weight of each subject verb object structure, as an support vector machine feature.

\begin{tabular}{|c|c|c|c|}
\hline Abuser onto victim & Weight of SVO ${ }^{\mathrm{a}}$ structure & Victim as subject & Weight of SVO structure \\
\hline convince & 0.95 & realize & 0.98 \\
\hline need & 0.94 & think & 0.91 \\
\hline isolate & 0.94 & !think & 0.91 \\
\hline promise & 0.92 & find & -0.88 \\
\hline love & 0.90 & learn & -0.88 \\
\hline !love & -0.89 & believe & 0.86 \\
\hline !hit & 0.89 & !know & 0.84 \\
\hline have & 0.87 & try & 0.80 \\
\hline leave & -0.80 & felt & 0.73 \\
\hline tell & 0.80 & know & -0.71 \\
\hline be & 0.78 & tell & 0.71 \\
\hline find & 0.76 & get & -0.70 \\
\hline choke & -0.75 & $N / A^{b}$ & N/A \\
\hline kill & -0.74 & N/A & N/A \\
\hline
\end{tabular}

${ }^{\mathrm{a}} \mathrm{SVO}$ : subject-verb-object.

${ }^{\mathrm{b}}$ N/A: not applicable.

Table 5 shows that physical abusers on victim verbs like choke and kill are associated with \#WhyILeft, whereas for the victim as subject verbs realize appears as the most indicative verb in the data, along with find and learn. Additionally, a predominance of verbs about cognitive manipulation appear in \#WhyIStayed tweets, such as convince, promise, believe, think, ! think (where "!" denotes negation, for example, I did not think he would...) and tell. Heise et al [19] suggested that emotional dependence and an optimistic hope for change are reasons for staying, which these manipulative verbs seem to corroborate. Other interesting findings are the equal and opposite effects of love and !love, and the verb!hit, which suggests that perhaps the narrators believed they were not experiencing abuse because it was not physical, or that they feared physical retribution if they tried to leave.

\section{Machine Learning on Linguistic Features}

We used naïve Bayes, logistic regression, linear SVM, and RBF SVM classifier methods to automatically predict whether a tweet was tagged with \#WhyIStayed or \#WhyILeft. The RBF SVM method performed slightly better than the others, achieving a maximum accuracy of $78 \%$ (SD 1\%) on the devset and 78\% on the test set using a subset of features and hyperparameters: $\max \mathrm{df}=12 \%, \mathrm{C}=10$, gamma $=1$. To better understand which linguistic features and preprocessing steps were most important to these classifiers, we performed feature ablation, following the procedure in Fraser et al [20], to determine the most important features the classifier used for prediction. Interestingly, the SVO features combined with ngrams worsened performance slightly, perhaps because of trigrams capturing the majority of SVO cases, but likely also because they just covered a small fraction of the dataset. The highest accuracy, nearly 
$78 \%$ on the test set, used a combination of ngrams and retweet counts for features and informal register (tone) replacement in the preprocessing step.

We then used the confidence score of the linear SVM (defined as the distance from the classifier's separating hyperplane in the feature space of the model) on each feature, taken as a single input to the SVM, as an estimate of that feature's discriminativeness, or ability to distinguish between the hashtag class labels (Tables 5 and 6). This method can be seen as an alternative to the ngram count, which measures the predictive power of each ngram, rather than its frequency [21].

Table 6. Top 10 features, with their linear support vector machine weights using ngrams and retweet counts as features, and informal register replacement during preprocessing. Except for try leave, the top features were all unigrams.

\begin{tabular}{llll}
\hline \#WhyIStayed & SVM $^{\mathrm{a}}$ weight & \#WhyILeft & SVM weight \\
\hline think & 3.0 & realize & 3.3 \\
believe & 1.6 & finally & 2.4 \\
convince & 1.6 & tired & 1.7 \\
tell & 1.5 & realise & 1.4 \\
say & 1.3 & daughter & 1.4 \\
try leave & 1.1 & son & 1.4 \\
money & 1.0 & die & 1.3 \\
abuser & 0.9 & strong & 1.3 \\
feel & 0.9 & kill & 1.2 \\
young & 0.9 & anymore & 1.2 \\
\hline
\end{tabular}

${ }^{\mathrm{a}} \mathrm{SVM}$ : support vector machine.

The SVM picked up on many of the same reasons for leaving and staying as those shown in Tables 3 and 4, but also revealed new ones, including tired, finally, and strong, which appear on the \#WhyILeft list (Table 6). These seem to suggest less an epiphany or triggering crisis and more a sense that the narrator was aware of and tolerated abuse for a long time until it became too much to bear.

For staying, language about cognitive and verbal manipulation was prominent (think, believe, convince, tell, say, and feel). Several new reasons also appeared: try leave, money, and young. The phrase try leave backs up claims in clinical literature that it is often difficult to gain external support to leave, and that victims of abuse frequently go through cycles of abuse that involve leaving and returning multiple times [19]. Financial distress is often a key factor for staying [19,22], so it is no surprise that money appears as a top feature for the SVM. The word young suggests that many were too young to leave or too naïve (due to their youth) to recognize that their relationship was abusive.

\section{Subject-Verb-Object Structures}

Restricting the dev- and test-sets to just those instances having an SVO structure, we trained the naïve Bayes, linear SVM, and RBF SVM. The linear SVM performed best, yielding 72\% accuracy.

Table 7 shows the top SVO structures using the confidence score of the linear SVM on each data item. Some interesting structures not found in Table 5 appear here. For example, the \#WhyILeft list reveals interventions from nonabusers (sister tell me). Taking a closer look at the supporting tweets, for example because my sorority sisters and roommates told me nothing about how he treated me was okay, suggests that these SVO structures refer to social support to which the victim has access. In the \#WhyIStayed class, church tell me once again shows that religious institutions can play a role in keeping victims in abusive relationships. Several tweets indicated that their church condoned abuse as a means of avoiding embarrassment and divorce, for example because the church told me that it was my responsibility as a godly wife to not embarrass him and just pray. 
Table 7. Top 10 subject-verb-object features for \#WhyIStayed and \#WhyILeft, with their support vector machine weights. An exclamation point (!) in front of a predicate verb indicates negation.

\begin{tabular}{llll}
\hline \#WhyIStayed & SVM $^{\mathrm{a}}$ weights & \#WhyILeft & SVM weights \\
\hline he hurt me & 1.1 & he tell him & 1.3 \\
they !remember him & 1.1 & he !protect me & 1.2 \\
he need me & 1.1 & he !tell me & 1.0 \\
he convince me & 1.1 & he lie me & 1.0 \\
she convince me & 1.1 & he stab me & 0.9 \\
he give child & 1.0 & he do kid & 0.89 \\
he remind me & 1.0 & sister tell me & 0.89 \\
he wear me & 1.0 & she have baby & 0.78 \\
he !abuse kid & 1.0 & he strangle me & 0.77 \\
church tell me & 0.99 & he attack me & \\
\hline
\end{tabular}

${ }^{\mathrm{a}} \mathrm{SVM}$ : support vector machine.

\section{Discussion}

\section{Principal Findings}

Our analysis shows that the process of leaving often involves a better understanding of the reality of an abusive relationship, or coming to terms with a long anticipated but hazardous decision to leave. The words and phrases found in our results tend to be about the pressures of staying versus leaving or the dynamics involved in leaving (Figure 2). Buel [22] explains why women may choose to stay in abusive relationships, including fear of retaliation, lack of financial independence, concern for their children, emotional dependence, lack of support from friends and family, fear of divorce and the potential to lose custody of their children, and/or an optimistic hope through love that their abuser will change. Children are often a factor in keeping victims in abusive relationships, and many victims will finally leave an abusive situation once their children have grown. The words and structures found in our results support many of these observations (eg, church tell me, emotional abuse, and daughter deserve better).

Heise et al [19] explain why victims of abuse leave and describe the dynamics of leaving. For instance, an increase in violence sometimes triggers a realization that their abuser will not change, that things are going to get worse, that the violence is going to affect their children, that they may be killed, etc. Support from friends, family, or society often allows those abused to leave. In any case, leaving is frequently a difficult process, involving cycles of denial, self-blame, and doubt. We found many of these same pressures and dynamics in our results (eg, realize love, want live, sister tell me). 
Figure 2. A pictorial summary of our results, grouped according to the forces that keep people in abusive relationships or cause them to leave and the dynamics involved in leaving. In the dynamics section, gray arrows denote pairs of textual features that represent opposing pressures and appeared on opposite lists in the same table (1-, 2-, or 3-gram, subject verb object, and support vector machine classification features). SVM: support vector machine; SVO: subject verb object.

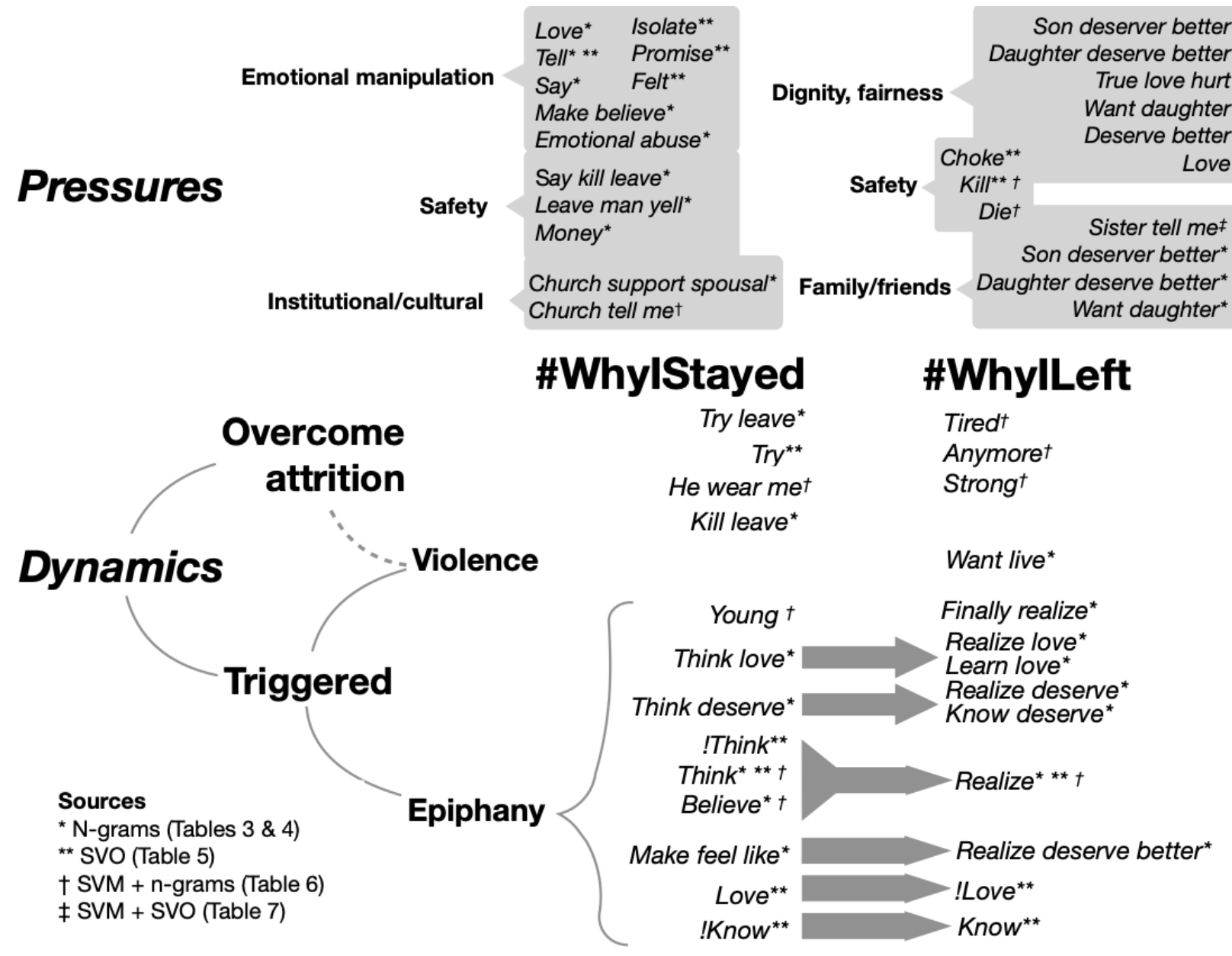

\section{Pressures for Staying Versus Leaving}

The main pressures we found for staying included emotional abuse, safety of self or other, and church communities. The pressures for leaving include a desire to be treated fairly or with dignity, the safety of self or other, and the concern for or support of a close family member.

The three pressures on each side interact with those on the other side in different ways. Emotional manipulation (a form of staying pressure) would seem to have less of an effect on victims as they become more aware of the injustice of their situation (leaving pressure). A sense of personal dignity (a leaving pressure) can cause victims to question the values of churches or other communities when they deny this dignity (a staying pressure).

For safety (both staying and leaving pressure), victims often say they stay in abusive relationships because they are afraid they will be physically or financially harmed if they leave, and they leave if they believe they or their children will be harmed or killed if they stay. Those who stay versus leave out of fear for personal safety are not necessarily the same individuals.

The last two pressures, church community and family or friends, are both about roles people outside the intimate partnership

play. There is a notable asymmetry between them, in that they represent different circles in a social ecology. Several researchers have noted disparities between how institutions and victims view IPV [23,24]. That church is the only community or institution specifically mentioned in our results is not too surprising, considering the importance of beliefs and knowledge on the dynamics of leaving a relationship and the role that churches play in preserving systems of beliefs. Lempert [13] discusses the pivotal roles that peer-level families and friends can play in helping victims leave. We found some evidence for this in our results, but the most frequent relationships mentioned were victims' children, who, as members of the family unit where the IPV occurred (and as frequent witnesses and victims themselves) were arguably in a different group from a peer-level family and friends, who were rarer in our results (Table 7).

\section{Dynamics of Leaving}

The dynamics of leaving can be roughly divided into two subgroups: those triggered by an event, such as an intervention by a friend, personal epiphany, or an imminent threat of harm, but before which the narrator was not expecting to leave, or even believed that their situation was unusually dire. The second group had to do with a slow wearing down, or attrition, where the victim was well aware of the harm the relationship was 
causing, but had to weigh those harms against the costs of leaving.

Of these groups, words and phrases related to event triggering, specifically epiphanies, seemed to be the most common. Most of these were generic, such as thought or realized. However, victims did mention that, before leaving, they failed to understand the nature of love or violence, or did not realize that the abuse they experienced was not normal. Community pressures sometimes played a role in reinforcing the feeling that the abuse was normal. Sometimes the birth or maturation of children causes the narrator to see themselves more objectively. Sometimes they even witnessed the abuse they experienced visited upon their children, and this triggered a deeper awareness of the grim reality of their situation.

Dynamics around violence typically involve the narrator at some point becoming aware, either slowly over time or by a sudden escalation that they are at imminent risk of serious harm to death if they remain in the relationship. Heise et al [19] suggested that victims of abuse leave after an increase in violence triggers a realization.

In attrition dynamics, either the cost of abuse begins to outweigh the cost of leaving, or the victim, after some period of weakness or disempowerment, is able to summon the strength to leave. Heise et al [19] suggest that women are often not passive victims of abuse. Instead, they actively attempt to maximize the safety of themselves and their children, while struggling in secret to navigate the (often insufficient) support structures available to them. Many victims return repeatedly to their abusers before leaving permanently [19]. In our data, direct evidence of this dynamic was scarce in the ngram analysis, but abundant in the SVM analysis (Tables 6 and 7). However, the fear of personal harm from leaving due to violence, impoverishment, etc. was a significant pressure in many cases, evidence of which was abundant in the ngram analysis (Tables 3 and 4).

\section{Ecological Model}

Many of the findings from this study support a four-level ecological model [25,26] proposed by Heise et al [19] and expanded on by the World Health Organization [27]. All four levels that increase the likelihood that a man will abuse his partner are found in these data to varying degrees.

\section{Individual}

Ngrams like hit and choke (acceptance of violence as a means of solving issues), childhood (experiencing or witnessing abuse as a child), and want daughter, son deserve better (trying to prevent their children from experiencing or witnessing abuse).

\section{Relationship}

Ngrams like money and financial (control of finances, economic stress) and the abuser onto victim verb !love (marital conflict)

\section{Community}

Ngrams like try leave and the abuser onto victim verb isolate (women's isolation), church support spousal, and church tell me (social groups that condone abuse).

\section{Societal}

Evidence for this last level was scarce; however, the SVO structure he need me suggests that abusers sometimes act out of frustration with societal norms or expectations.

\section{Why Did Both \#WhyIIStayed and \#WhyILeft Go Viral?}

Our results fall short of spelling out exactly why both the \#WhyIStayed and \#WhyILeft tags went viral, but they do paint a rich picture of the pressures and dynamics involved in staying versus leaving abusive relationships. Certainly, many reasonable explanations for the virality of these hashtags may have nothing to do with the actual stories of abuse disclosed. Perhaps in reaction to \#WhyIStayed (which was tweeted first) activists adopted \#WhyILeft to send a message that was more upbeat and empowering than \#WhyIStayed. Certainly, the shift from emotional abuse to self-dignity and fairness comes with a shift from passivity to a more active and empowered role. One fairly clear, consistent pattern that we observed in our results was that leaving involved significant changes in life circumstances. Perhaps it is difficult even to recall the frame of mind one was in on either side of the leaving event without some kind of framing device like a hashtag, or perhaps having one hashtag for each side of this transition emphasizes the importance and significance of the transition itself in a way that having only one hashtag in the public sphere does not.

\section{Limitations}

We note several limitations in this research.

\section{Bias Toward Female Victims and Male Abusers}

We had hoped to study gender as a discriminative factor; however, instances with certain female abusers were rare (approximately 230 instances). Although it was difficult to determine a certain number, it appeared that the vast majority of the victims were female. This may be in part because males have significant inhibitions in reporting their abuse [8] and may therefore be less likely to tweet about their experiences and make their narratives public. It could also be that men do not face the same obstacles to leaving an abusive relationship, for instance, due to access to finances and/or alternative housing. Furthermore, we had no ability to stratify the data by sexual orientation, which could have implications for staying or leaving an abusive relationship for those that identify as lesbian, gay, transgender, or bisexual. There is a need for more research in this area.

\section{Unique and/or Rare Forms of Abuse Missing}

The properties of abuse and reasons for staying and leaving discovered in these data are affected by their relative frequency of occurrence. Unique and/or rare reasons for staying and leaving, and rare aspects of abusive relationships, may not be discovered using the methods presented here.

\section{Noise}

As with most social media data, it is important to know that these datasets likely contain posts by spam bots, lies by the users, or jokes that were missed by filters. 


\section{Handcrafted Pronouns and Lexical Items}

The pronouns and lexical items used to convert the SVO features to abuser onto victim structures were handcrafted, potentially restricting the discriminative verbs that appear in the Results section.

\section{Preprocessing}

Lowercasing, stoplisting, and lemmatizing help to reduce dimensionality and thus improve learning performance, but case, tense, and certain ngrams that appear in the stoplist may be important features that were missed due to these preprocessing steps.

\section{Length of Text}

Although we recognize that these narratives are brief, qualitative methods such as free listing [28] often make use of brief texts. The notion of using words to create conceptual frameworks is not uncommon in mixed-method research. We suggest that our findings are worthy of continued, future exploration.

More broadly, our results show that when social media presents a large amount of data on a subject like IPV, even simple statistics such as ngrams can reveal a great deal of information about the nature of the subject. Machine learning methods, with their biases toward specific decision-making outcomes, reveal different insights. Although none of these approaches yielded effective predictive models, they provided data that were qualitative and quantitative enough to lend support to existing theories of IPV.

\section{Comparison With Prior Work}

Computational methods are applied to better understand the \#WhyIStayed/\#WhyILeft movement. Recent studies of this movement are concurrent with ours [29,30]. However, they were about their historical and qualitative aspects, or were based on a tiny sample of the data available. Our work is complementary to these studies, and our goal was to provide quantitative results that lend insight and credibility to these prior qualitative and clinical observations.

An extensive body of work explores how to extract affective information and other subjective signals from social media [31-35]. Adding part of speech tags to ngrams is often attempted as well as creating word classes via data inspection, using morphosyntactic features, and exploiting the sentiment of text instances. For instance, in Xu et al [36], linear models with ngrams are recommended for their simplicity and high accuracy, although in Lamb et al [37], word classes, Twitter-specific stylometry (retweet counts, hashtags, user mentions, and emoticons), and an indicator for phrases beginning with a verb were found to be helpful over ngrams on two different tasks.

Many of these works are motivated by commercial applications, for example, mining to extract individuals' sentiments about products or services [38,39]. Another stream of research focuses specifically on modeling, extracting, and/or tracking emotions on social media. Some of these works deal with emotions independent of context [40-42]. Other studies have studied their correlations with time $[43,44]$ or other socioeconomic phenomena [45]. Still others model emotion as a social contagion [46-48] or focus on specific contexts, such as employment [49]. More recently, researchers have focused on specific emotional conditions or behavioral phenomena.

With respect to behavioral health, Coppersmith et al [50-52] built classifiers for detecting a number of mental health conditions, including major depression, posttraumatic stress disorder, seasonal affective disorder, and attention deficit hyperactivity disorder, by training tweets that match regular expressions related to each condition. De Choudhury et al $[53,54]$ collected labels to consider as ground truth regarding the presence of major depression using crowd sourcing. Other researchers have focused on specific health issues, including posttraumatic stress disorder [51], early detection of epidemics $[37,55]$, and bullying tweets $[36,56]$. A number of recent papers have studied suicidality on social media [57,58], or risk factors for suicide such as distress [59]. Closer to this paper and concurrent with our research, sexual abuse disclosures via anonymous Reddit were studied qualitatively by O'Neill [33] and quantitatively by Andalibi et al [60]. Subramani et al [61] performed a similar quantitative analysis of Facebook pages related to domestic violence. Karlekar and Bansal [10] studied the use of machine learning to extract narratives of sexual harassment from the SafeCity web-based forum.

\section{Conclusions}

The research presented here demonstrates the power of social media to uncover meaningful structural, semantic, linguistic, and textual characteristics, including actions, stakeholders, and situations related to abusive relationships. It revealed micronarratives in tweeted reasons for staying versus leaving abusive relationships. A classifier for distinguishing between tweeted reasons for staying versus leaving abusive relationships achieved an accuracy of $78 \%$. Our textual analysis, in showing that partners leave violent relationships after an epiphany of self-realization, is validated in the clinical literature. Moreover, the sheer volume of data present in social media suggests the potential to learn more details about the nature and dynamics of interpersonal violence than-due to the stigma and shame related to disclosing stories of victimization that the \#WhyIStayed/\#WhyILeft movement has helped erode-are currently known and may potentially help clinicians to reduce the harm caused by abusive relationships.

There are a number of interesting directions for future work. For instance, social media data with course-grained geotags could be used to study whether reasons for staying and leaving differ across geographical locations, or how varying community-level characteristics of those locations (eg, poverty level, population density, education levels, etc.) affect IPV victims. Analysis of web abuse discourse across varied media would strengthen the present findings if they overlapped, and perhaps lead to a better understanding of how victims make sense of and manage IPV and abuse. 


\section{Acknowledgments}

This work was supported in part by a Golisano College of Computing and Information Sciences Kodak Endowed Chair Fund Health Information Technology Strategic Initiative Grant and National Science Foundation Award \#SES1111016. This study was also supported in part by the Susan B. Anthony Center at the University of Rochester.

\section{Conflicts of Interest}

None declared.

\section{References}

1. Straus H, Cerulli C, McNutt LA, Rhodes KV, Conner KR, Kemball RS, et al. Intimate partner violence and functional health status: associations with severity, danger, and self-advocacy behaviors. J Womens Health (Larchmt) 2009 May;18(5):625-631 [FREE Full text] [doi: 10.1089/jwh.2007.0521] [Medline: 19445614]

2. Straus H, Cerulli C, McNutt LA, Rhodes KV, Conner KR, Kemball RS, et al. Intimate partner violence and functional health status: associations with severity, danger, and self-advocacy behaviors. J Womens Health (Larchmt) 2009 May;18(5):625-631 [FREE Full text] [doi: 10.1089/jwh.2007.0521] [Medline: 19445614]

3. Dichter ME, Cerulli C, Bossarte RM. Intimate partner violence victimization among women veterans and associated heart health risks. Womens Health Issues 2011;21(4 Suppl):S190-S194. [doi: 10.1016/j.whi.2011.04.008] [Medline: 21724140]

4. Pigeon WR, Cerulli C, Richards H, He H, Perlis M, Caine E. Sleep disturbances and their association with mental health among women exposed to intimate partner violence. J Womens Health (Larchmt) 2011 Dec;20(12):1923-1929 [FREE Full text] [doi: 10.1089/jwh.2011.2781] [Medline: 21988551]

5. Cerulli C, Poleshuck E, Raimondi C, Veale S, Chin N. "What fresh hell is this?" victims of intimate partner violence describe their experiences of abuse, pain, and depression. J Fam Violence 2012 Nov;27(8):773-781 [FREE Full text] [doi: 10.1007/s10896-012-9469-6] [Medline: 23226694]

6. Ard KL, Makadon HJ. Addressing intimate partner violence in lesbian, gay, bisexual, and transgender patients. J Gen Intern Med 2011 Aug;26(8):930-933 [FREE Full text] [doi: 10.1007/s11606-011-1697-6] [Medline: 21448753]

7. Whitfield C, Anda R, Dube S, Felitti V. Violent childhood experiences and the risk of intimate partner violence in adults. J Interpers Violence 2016 Jul 2;18(2):166-185 [FREE Full text] [doi: 10.1177/0886260502238733]

8. Smith SG, Chen J, Basile KC, Gilbert LK, Merrick MN, Patel N, et al. The National Intimate Partner and Sexual Violence Survey (NISVS): 2010-2012 State Report. National Center for Injury Prevention and Control, Centers for Disease Control and Prevention. 2017. URL: https://www.cdc.gov/violenceprevention/pdf/NISVS-StateReportBook.pdf [accessed 2020-10-17]

9. Burge SK, Becho J, Ferrer RL, Wood RC, Talamantes M, Katerndahl DA. Safely examining complex dynamics of intimate partner violence. Fam Syst Health 2014 Sep;32(3):259-270. [doi: 10.1037/fsh0000013] [Medline: 24911769]

10. Kalekar S, Bansal M. Understanding Diverse Forms of Sexual Harassment Personal Stories. In: Proceedings of the 2018 Conference on Empirical Methods in Natural Language Processing. 2018 Presented at: EMNLP'18; October 31-November 4, 2018; Brussels, Belgium p. 2805-2811. [doi: 10.18653/v1/d18-1303]

11. Bien L. A Complete Timeline of the Ray Rice Assault Case. SBnation: Sports News, Video, Live Coverage, Community. 2014. URL: https://www.sbnation.com/nfl/2014/5/23/5744964/ray-rice-arrest-assault-statement-apology-ravens [accessed 2019-06-03] [WebCite Cache ID 78rLH91Yb]

12. Bahadur N. \#WhyIStayed Stories Reveal Why Domestic Violence Survivors Can't 'Just Leave'. HuffPost India: Breaking News, Entertainment, Tech, Lifestyle. 2014. URL: https://www.huffpost.com/entry/ whyistayed-twitter-domestic-violence n 5790320 [accessed 2019-06-03] [WebCite Cache ID 78rKJ2ZVO]

13. Lempert L. The other side of help: negative effects in the help-seeking processes of abused women. Qual Sociol 1997;20(2):289-309. [doi: 10.1023/A:1024769920112]

14. Tifft L. Battering Of Women: The Failure Of Intervention And The Case For Prevention. Boulder, CO: Westview Press; 1993.

15. Varoquaux G, Buitinck L, Louppe G, Grisel O, Pedregosa F, Mueller A. Scikit-learn: machine learning in python. GetMobile: Mobile Comp and Comm 2015 Jun;19(1):29-33. [doi: 10.1145/2786984.2786995]

16. Greff K, Srivastava RK, Koutnik J, Steunebrink BR, Schmidhuber J. LSTM: a search space odyssey. IEEE Trans Neural Netw Learn Syst 2017 Oct;28(10):2222-2232. [doi: 10.1109/TNNLS.2016.2582924] [Medline: 27411231]

17. Karpathy A. Char-RNN: Multi-Layer Recurrent Neural Networks (LSTM, GRU, RNN). GitHub: Where the World Builds Software. URL: https://github.com/karpathy/char-rnn [accessed 2019-06-03] [WebCite Cache ID 78rQNt2XR]

18. Warrens M. Inequalities between multi-rater kappas. Adv Data Anal Classif 2010 Oct 10;4(4):271-286 [FREE Full text] [doi: $10.1007 / \mathrm{s} 11634-010-0073-4]$

19. Heise L, Ellsberg M, Gottemoeller M. Ending violence against women. Popul Rep L 1999 Dec(11):1-43 [FREE Full text] [Medline: 11056940$]$

20. Fraser K, Hirst G, Graham N, Meltzer J, Black S, Rochon E. Comparison of Different Feature Sets for Identification of Variants in Progressive Aphasia. In: Proceedings of the Workshop on Computational Linguistics and Clinical Psychology: 
From Linguistic Signal to Clinical Reality. 2014 Presented at: CLPsych'14; June 27, 2014; Baltimore, MD. [doi: $10.3115 / \mathrm{v} 1 / \mathrm{w} 14-3203]$

21. Guyon I, Weston J, Barnhill S, Vapnik V. Gene selection for cancer classification using support vector machines. Mach Learn 2002;46(1-3):389-422 [FREE Full text] [doi: 10.1023/a:1012487302797]

22. Buel S. Fifty obstacles to leaving, aka, why abuse victims stay. Colo Law 1999;28(10):19-28 [FREE Full text]

23. Garcia-Moreno C, Watts C, Heise L. Putting Women First: Ethical and Safety Recommendations for Research on Domestic Violence Against Women. Department of Gender and Womens Health, World Health Organization. 2001. URL: https:/ /www.who.int/gender/violence/womenfirtseng.pdf [accessed 2020-10-19]

24. Liang B, Goodman L, Tummala-Narra P, Weintraub S. A theoretical framework for understanding help-seeking processes among survivors of intimate partner violence. Am J Community Psychol 2005 Sep;36(1-2):71-84. [doi: 10.1007/s10464-005-6233-6] [Medline: 16134045]

25. Bronfenbrenner U. The Ecology of Human Development. Cambridge, MA: Harvard University Press; 2009.

26. Heise LL. Violence against women: an integrated, ecological framework. Violence Against Women 1998 Jun;4(3):262-290. [doi: 10.1177/1077801298004003002] [Medline: 12296014]

27. Krug EG, Mercy JA, Dahlberg LL, Zwi AB. The world report on violence and health. Lancet 2002 Oct 5;360(9339):1083-1088. [doi: 10.1016/S0140-6736(02)11133-0] [Medline: 12384003]

28. Quinlan M. The freelisting method. In: Liamputtong P, editor. Handbook of Research Methods in Health Social Sciences. Singapore: Springer Singapore; 2017.

29. Cravens J, Whiting J, Aamar R. Why I stayed/left: an analysis of voices of intimate partner violence on social media. Contemp Fam Ther 2015 Sep 25;37(4):372-385 [FREE Full text] [doi: 10.1007/s10591-015-9360-8]

30. Guidry J. Tweeting \#whyileft and \#whyistayed: Analyzing the Conversation Around Intimate Partner Violence on Twitter. In: Proceedings of the 143rd American Public Health Association Annual Meeting and Exposition. 2015 Presented at: APHA'15; October 31-November 4, 2015; Chicago, IL.

31. Pak A, Paroubek P. Twitter as a Corpus for Sentiment Analysis and Opinion Mining. In: Proceedings of the International Conference on Language Resources and Evaluation. 2010 Presented at: LREC'10; May 17-23, 2010; Valleta, Malta URL: https://www.researchgate.net/publication/220746311 Twitter as a Corpus for Sentiment Analysis and Opinion Mining

32. Wang W, Chen L, Thirunarayan K, Sheth A. Harnessing Twitter 'Big Data' for Automatic Emotion Identification. In: International Conference on Privacy, Security, Risk and Trust and International Conferenece on Social Computing. 2012 Presented at: ASE/IEEE'12; September 3-5, 2012; Amsterdam, Netherlands p. 587-592. [doi: 10.1109/socialcom-passat.2012.119]

33. O'Neill T. 'Today I Speak': Exploring How Victim-Survivors Use Reddit. Int J Crime Justice Soc Democr 2018 Mar 1;7(1):44-59 [FREE Full text] [doi: 10.5204/ijcjsd.v7i1.402]

34. Kim S, Bak J, Oh A. Do You Feel What I Feel? Social Aspects of Emotions in Twitter Conversations. In: Proceedings of the 6th AAAI International Conference on Weblogs and Social Media. 2012 Presented at: AAAI'12; June 4-7, 2012; Dublin, Ireland.

35. Hannak A, Anderson E, Barrett L, Lehmann S, Mislove A, Riedewald M. Tweetin' in the Rainxploring Societal-scale Effects of Weather on Mood. In: Proceedings of the 6th AAAI International Conference on Weblogs and Social Media. 2012 Presented at: AAAI'12; June 4-7, 2012; Dublin, Ireland.

36. Xu JM, Jun KS, Zhu X, Bellmore A. Learning From Bullying Traces in Social Media. In: Proceedings of the 2012 Conference of the North American Chapter of the Association for Computational Linguistics: Human Language Technologies. 2012 Presented at: NAACL HLT'12; June 3-8, 2012; Montreal, Canada. [doi: 10.5555/2382029.2382139]

37. Lamb A, Paul M, Dredze M. Separating Fact From Fear: Tracking Flu Infections on Twitter. In: Proceedings of the Conference of the North American Chapter of the Association for Computational Linguistics: Human Language Technologies. 2013 Presented at: NAACl'13; June 9-14, 2013; Atlanta, GA.

38. Thelwall M, Buckley K, Paltoglou G. Sentiment in Twitter events. J Am Soc Inf Sci 2010 Dec 6;62(2):406-418 [FREE Full text] [doi: 10.1002/asi.21462]

39. Pfitzner R, Garas A, Schweitzer F. Emotional Divergence Influences Information Spreading in Twitter. In: Proceedings of the 6th AAAI International Conference on Weblogs and Social Media. 2012 Presented at: AAAI'12; June 4-7, 2012; Dublin, Ireland.

40. Mohammad S. \#Emotional Tweets. In: Proceedings of the First Joint Conference on Lexical and Computational Semantics. 2012 Presented at: SemEval'12; June 7-8, 2012; Montreal, Canada. [doi: 10.5555/2387636.2387676]

41. De Choudhury M, Gamon M, Counts S. Happy, nervous or surprised? Classification of human affective states in social media. In: Proceedings of the 6th AAAI International Conference on Weblogs and Social Media. 2012 Presented at: AAAI'12; June 4-7, 2012; Dublin, Ireland p. 435-438.

42. De Choudhury M, Counts S, Gamon M. Not All Moods re Created Equal! A Exploring Human Emotional States in Social Media. In: Proceedings of the 6th AAAI International Conference on Weblogs and Social Media. 2012 Presented at: AAAI'12; June 4-7, 2012; Dublin, Ireland.

43. Golder SA, Macy MW. Diurnal and seasonal mood vary with work, sleep, and daylength across diverse cultures. Science 2011 Sep 30;333(6051):1878-1881. [doi: 10.1126/science.1202775] [Medline: 21960633] 
44. Dodds PS, Harris KD, Kloumann IM, Bliss CA, Danforth CM. Temporal patterns of happiness and information in a global social network: hedonometrics and Twitter. PLoS One 2011;6(12):e26752 [FREE Full text] [doi: 10.1371/journal.pone.0026752] [Medline: 22163266]

45. Bollen J, Pepe A, Mao H. Modeling Public Mood and Emotion: Twitter Sentiment and Socio-economic Phenomena. In: Proceedings of the Fifth International AAAI Conference on Weblogs and Social Media. 2011 Presented at: AAAI'11; July 17-21, 2011; Barcelona, Spain p. 450-453.

46. Bollen J, Gonçalves B, Ruan G, Mao H. Happiness is assortative in online social networks. Artif Life 2011;17(3):237-251. [doi: 10.1162/artl a 00034] [Medline: 21554117]

47. Kivran-Swaine F, Naaman M. Network Properties and Social Sharing of Emotions in Social Awareness Streams. In: Proceedings of the ACM Conference on Computer Supported Cooperative Work. 2011 Presented at: ACM Conference on Computer Supported Cooperative Work and March; March 2011; Hangzhou, China p. 379-382. [doi: $\underline{10.1145 / 1958824.1958882]}$

48. Kramer ADI, Guillory JE, Hancock JT. Experimental evidence of massive-scale emotional contagion through social networks. Proc Natl Acad Sci U S A 2014 Jun 17;111(24):8788-8790. [doi: 10.1073/pnas.1320040111] [Medline: 24889601]

49. De Choudhury M, Counts S. Understanding Affect in the Workplace via Social Media. In: Proceedings of the 16th ACM Conference on Computer Supported Cooperative Work and Social Media. 2013 Presented at: CSCW'13; February, 2013; San Antonio, Texas p. 303-316. [doi: 10.1145/2441776.2441812]

50. Coppersmith G, Dredze M, Harman C. Quantifying Mental Health Signals in Twitter. In: Proceedings of the Workshop on Computational Linguistics and Clinical Psychology. 2014 Presented at: CLCP'14; June 27, 2014; Baltimore, Maryland p. 51-60. [doi: 10.3115/v1/w14-3207]

51. Coppersmith G, Harman C, Dredze M. Measuring Post Traumatic Stress Disorder in Twitter. In: Proceedings of the 8th AAAI International Conference on Weblogs and Social Mediaq. 2014 Presented at: ICWSM'14; June 1-4, 2014; Ann Arbor,

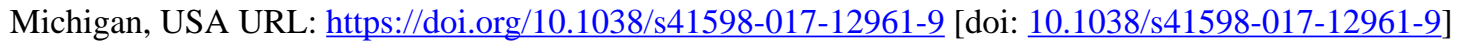

52. Coppersmith G, Dredze M, Harman C, Hollingshead K. From ADHD to SAD: Analyzing the Language of Mental Health on Twitter Through Self-reported Diagnoses. In: Proceedings of the Workshop on Computational Linguistics and Clinical Psychology. 2015 Presented at: CLCP'15; June 5, 2015; Denver, CO p. -10. [doi: 10.3115/v1/w15-1201]

53. De Choudhury M, Counts S, Horvitz E. Social Media as a Measurement Tool of Depression in Populations. In: Proceedings of the 5th ACM International Conference on Web Science. 2013 Presented at: 5th ACM International Conference on Web Science; May 2-4, 2013; Paris, France p. 47-56. [doi: 10.1145/2464464.2464480]

54. De Choudhury M, Gamon M, Counts S, Horvitz E. Predicting depression via social media. In: Proceedings of the 7th AAAI International Conference on Weblogs and Social Media. 2013 Presented at: AAAI'13; July 8-11, 2013; Cambridge, MA p. 128-137.

55. Velardi P, Stilo G, Tozzi AE, Gesualdo F. Twitter mining for fine-grained syndromic surveillance. Artif Intell Med 2014 Jul;61(3):153-163. [doi: 10.1016/j.artmed.2014.01.002] [Medline: 24613716]

56. Xu JM, Burchfiel B, Zhu X, Bellmore A. An Examination of Regret in Bullying Tweets. In: Proceedings of the 2013 Conference of the North American Chapter of the Association for Computational Linguistics: Human Language Technologies. 2013 Presented at: NAACL HLT'13; June 9-13, 2013; Atlanta, GA p. 697-702.

57. Burnap P, Colombo G, Amery R, Hodorog A, Scourfield J. Multi-class machine classification of suicide-related communication on Twitter. Online Soc Netw Media 2017 Aug;2:32-44 [FREE Full text] [doi: 10.1016/j.osnem.2017.08.001] [Medline: 29278258]

58. Luxton DD, June JD, Fairall JM. Social media and suicide: a public health perspective. Am J Public Health 2012 May; 102 Suppl 2:S195-S200. [doi: 10.2105/AJPH.2011.300608] [Medline: 22401525]

59. Homan C, Johar R, Liu T, Lytle M, Silenzio V, Alm C. Analyzing Fine-grained Distress at Scale. In: Proceedings of the Workshop on Computational Linguistics and Clinical Psychology. 2014 Presented at: CLPsych'14; June 27, 2014; Baltimore, MD p. 107-117. [doi: $10.3115 / \mathrm{v} 1 / \mathrm{w} 14-3213$ ]

60. Andalibi N, Haimson O, de Choudhury M, Forte A. Understanding Social Media Disclosures of Sexual Abuse Through the Lenses of Support Seeking and Anonymity. In: Proceedings of the CHI Conference on Human Factors in Computing Systems. 2016 Presented at: CHI'16; May 7-12, 2016; San Jose, CA p. 3906-3918. [doi: 10.1145/2858036.2858096]

61. Subramani S, Vu HQ, Wang H. Intent Classification Using Feature Sets for Domestic Violence Discourse on Social Media. In: Proceedings of the 4th Asia-Pacific World Congress on Computer Science and Engineering. 2017 Presented at: APWC on CSE'17; Dec 11-13, 2017; Mana Island, Fiji p. 129-136 URL: https://arxiv.org/abs/1804.03497 [doi: 10.1109/apwconcse.2017.00030]
Abbreviations
IPV: intimate partner violence
RBF: radial basis function
SVM: support vector machine
SVO: subject-verb-object 


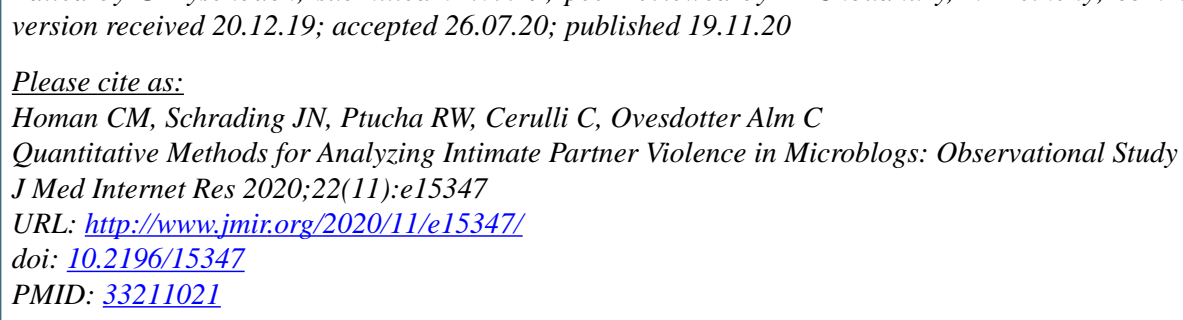

(OChristopher Michael Homan, J Nicolas Schrading, Raymond W Ptucha, Catherine Cerulli, Cecilia Ovesdotter Alm. Originally published in the Journal of Medical Internet Research (http://www.jmir.org), 19.11.2020. This is an open-access article distributed under the terms of the Creative Commons Attribution License (https://creativecommons.org/licenses/by/4.0/), which permits unrestricted use, distribution, and reproduction in any medium, provided the original work, first published in the Journal of Medical Internet Research, is properly cited. The complete bibliographic information, a link to the original publication on http://www.jmir.org/, as well as this copyright and license information must be included. 